Research Article

\title{
Bioinformatics-Based Identification of lncRNA-miRNA-mRNA Network in Dilated Cardiomyopathy and Drug Prediction
}

\author{
Wei Liu $\mathbb{D},{ }^{1}$ Jinqiang Cai $\mathbb{D}^{2}{ }^{2}$ Mengjie Tang, ${ }^{3}$ and QinJing Yang $\mathbb{D}^{1}$ \\ ${ }^{1}$ Department of Pharmacy, The Third Xiangya Hospital, Central South University, Changsha 410013, China \\ ${ }^{2}$ Department of Pharmacy, Xiangya Medical College, Central South University, Changsha 410013, China \\ ${ }^{3}$ Hunan Cancer Hospital, The Affiliated Cancer Hospital of Xiangya School of Medicine, Central South University, \\ Changsha 410013, China
}

Correspondence should be addressed to QinJing Yang; yangqinjing0624@163.com

Received 27 January 2021; Revised 4 February 2021; Accepted 17 February 2021; Published 4 March 2021

Academic Editor: Tingting Hong

Copyright $\odot 2021$ Wei Liu et al. This is an open access article distributed under the Creative Commons Attribution License, which permits unrestricted use, distribution, and reproduction in any medium, provided the original work is properly cited.

\begin{abstract}
Background. Dilated cardiomyopathy (DCM) is a cardiovascular disease of unknown etiology with progressive aggravation. More and more studies have shown that long noncoding RNAs (lncRNAs) play an essential role in dilated cardiomyopathy formation and development. The mechanism of action of competitive endogenous RNA (ceRNA) networks formed based on the principle that IncRNAs affect mRNAs' expression level by competitively binding microRNAs (miRNAs) in dilated cardiomyopathy has rarely been reported. Objective. This study is aimed at constructing a lncRNA-miRNA-mRNA ceRNA network by bioinformatics analysis methods, discovering, and validating potential biomarkers of DCM in the ceRNA network and determining possible therapeutic targets from them for drug prediction. Methods. A lncRNA dataset and a mRNA microarray dataset were downloaded from the Gene Expression Omnibus Database (GEO). Gene expression was compared between blood samples from patients with dilated cardiomyopathy and blood samples from normal subjects to identify differential expression of lncRNAs and mRNAs. The lncRNA-miRNA-mRNA network was constructed using bioinformatics tools, and functional and pathway enrichment analysis and protein-protein interactions were performed. The mRNAs in the network and the proteins they encode are then used as targets for predicting drugs. Besides, the expression of lncRNAs in the ceRNA network was validated by real-time quantitative PCR (qRT-PCR) experiments in vitro. Results. The differentially expressed lncRNA-miRNAmRNA ceRNA network in dilated cardiomyopathy was successfully established. Two differentially overexpressed key lncRNAs were found from the network: AC093817 and AC091062, and qRT-PCR experiments further validated the overexpression of AC093817 and AC091062. The mRNAs in the network and the proteins encoded by the mRNAs were used for drug prediction to get related drugs. Conclusion. This study supports a possible mechanism and drug development of dilated cardiomyopathy, AC093817 and AC091062 being potential biomarkers of dilated cardiomyopathy.
\end{abstract}

\section{Introduction}

Dilated cardiomyopathy is defined as left ventricular dilatation and left ventricular systolic dysfunction in the absence of abnormal load (hypertension, valvular disease) or coronary artery disease sufficient to cause global systolic function impairment [1]. Risk factors for dilated cardiomyopathy include genetic and environmental factors. Dilated cardiomyopathy may present with autosomal dominant, monogenic features with $\mathrm{X}$ chromosome, autosomal recessive inheritance, or with the effects of environmental factors such as nutritional deficiency, endocrine dysfunction, and taking cardiotoxic drugs such as Adriamycin [1,2]. The early symptoms of dilated cardiomyopathy are not obvious, and many patients have progressed to the terminal stage when they are diagnosed with the disease, which can lead to heart failure or even death [3]. Therefore, new biomarkers are essential for the early diagnosis and prognosis of dilated cardiomyopathy. Although previous studies have found that genetic factors play a crucial role in dilated cardiomyopathy, the pathogenesis of dilated cardiomyopathy is still unclear, and new treatment strategies are lacking. 


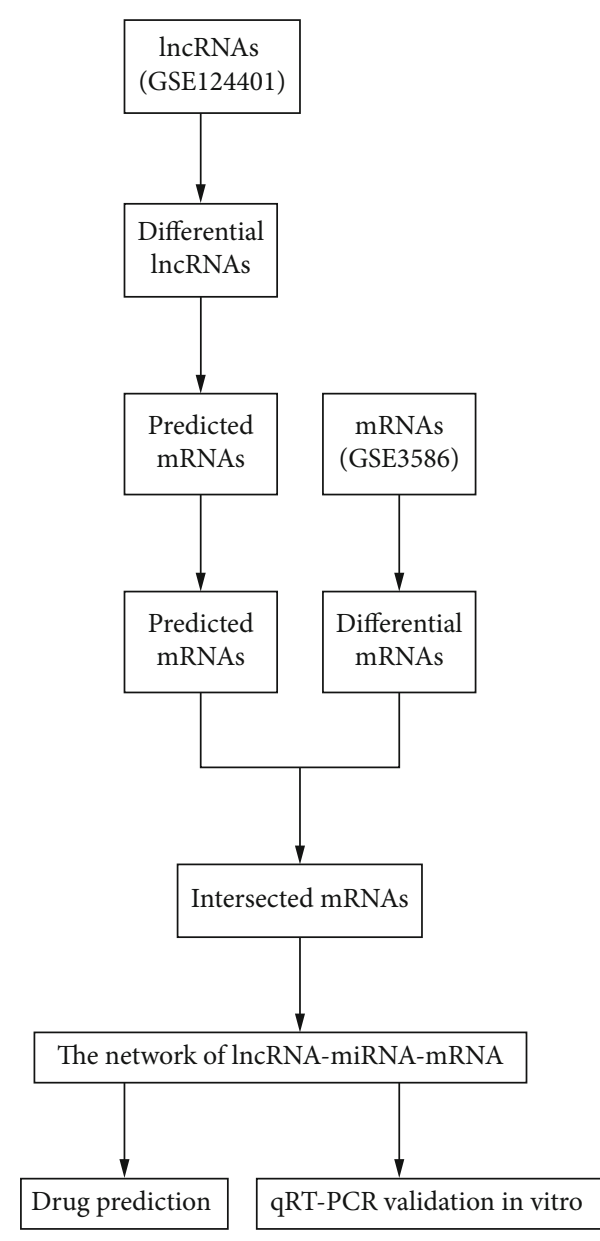

(a)

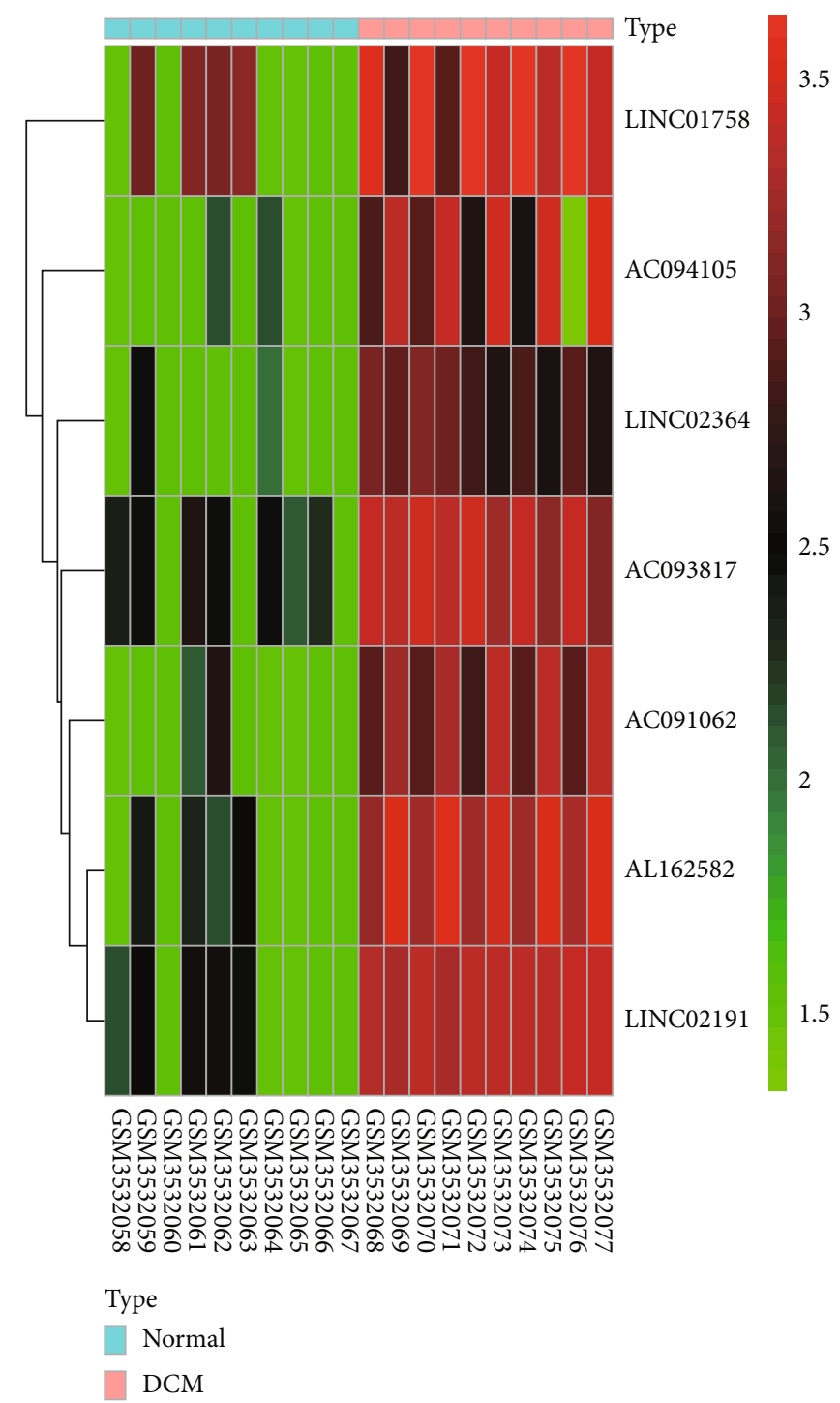

(b)

FIgure 1: Continued. 


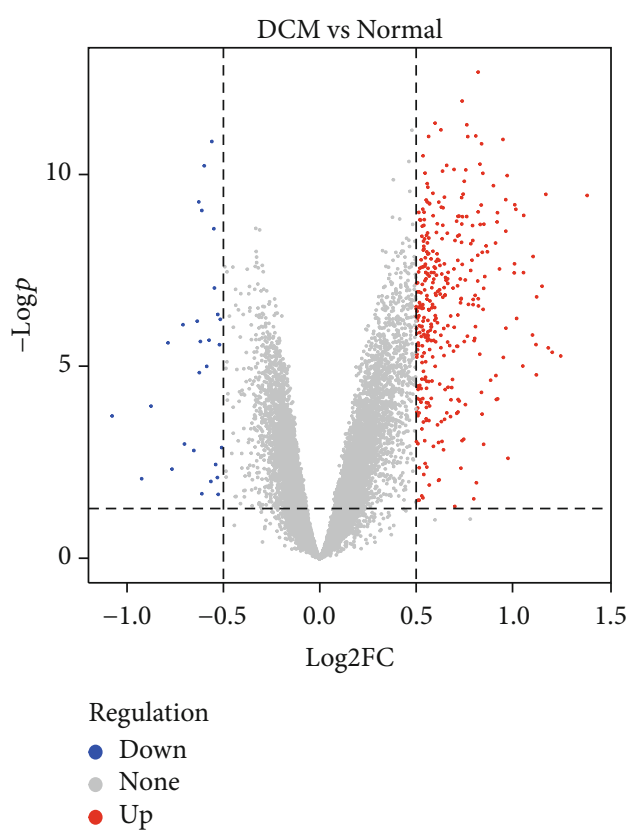

(c)

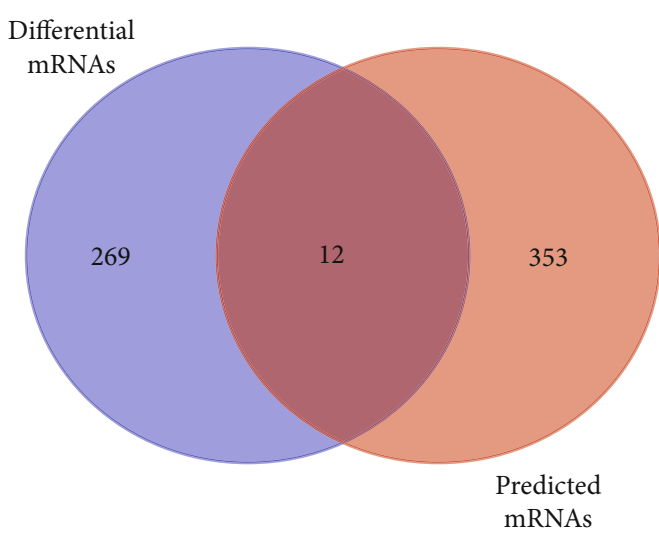

(d)

FIGURE 1: Flow chart of the approach and identification of intersected mRNAs in DCM (dilated cardiomyopathy). (a) Flow chart of the approach utilized in this study. (b) The heatmap of differentially expressed lncRNAs from the GSE124401 dataset. (c) The volcano plot of differentially expressed mRNAs from GSE3586 dataset. (d) The intersection analysis of predicted mRNAs and differential mRNAs.

lncRNAs are a group of RNAs > 200 nucleotides in length, without protein-coding function, regulating gene expression at the epigenetic, transcriptional, and posttranscriptional levels participating actively in various physiological and pathological processes $[4,5]$. In recent years, more and more evidence has shown that lncRNAs play a vital role in the occurrence and development of dilated cardiomyopathy and are very critical biomarkers and therapeutic targets. Li et al. found the most upregulated lncRNA RP11-544D21.2 in DCM patients, and this human-specific lncRNA significantly regulated DCM-related genes in cardiomyocytes and affected the ductal formation and cell migration in endothelial cells [6]. Lin et al. found that differentially expressed lncRNAs are involved in some specific biological processes and regulate some signaling pathways in the plasma of heart failure patients with dilated cardiomyopathy and ischemic cardiomyopathy [7]. Wang et al. predicted a novel function of an annotated lncRNA-H19 that may regulate apoptotic signal-regulated kinases through pathway analysis of 39 key $\operatorname{lncRNAs}$ that regulate key pathways in myocardial infarction [8]. Cheng and Jiang found that IncRNA HAND2-AS1 may be involved in end-stage dilated cardiomyopathy [3].

Luo et al. identified IDI2-AS1 and XIST two lncRNAs and their associated pathways in the pathogenesis of DCM, providing potential targets for the diagnosis and treatment of DCM [9]. Zhang et al. found that circulating lncRNA ENST00000507296 is a biomarker for the prognosis of DCM patients [10]. Qiu et al. found that AC061961.2, LING01-AS1, and RP11-13E1.5 were downregulated in DCM patients' myocardial tissue, and these lncRNAs could be used as critical diagnostic biomarkers and therapeutic targets for DCM [11]. Zhang et al. found that the H19/miR-675 axis was involved in the promoting effect of cardiomyocyte apoptosis by targeting PA2G4, providing a new therapeutic strategy for treating doxorubicin-induced DCM [5]. Tao et al. identified four lncRNA-miRNA pairs associated with DCM, which can be used as candidate diagnostic biomarkers or potential therapeutic targets for DCM [12]. However, the mechanism of action of lncRNA-associated ceRNA networks in dilated cardiomyopathy is unknown. Therefore, it is necessary to construct a lncRNA-miRNA-mRNA ceRNA network to obtain more information about the treatment and diagnosis of dilated cardiomyopathy from the ceRNA network.

The aim of this study was to identify differentially expressed lncRNAs and mRNAs using the limma package in $\mathrm{R}$ software. miRNAs were predicted using the miRcode database by differentially expressed lncRNAs, mRNAs were predicted by the combination of Targetscan, miRTarBase, and miRDB databases, and finally, differentially expressed mRNAs and predicted mRNAs were intersected to construct a lncRNAmiRNA-mRNA ceRNA network by Cytoscape software. mRNAs in the network were subjected to GO enrichment analysis, KEGG enrichment analysis, and construction of proteinprotein interaction network. The lncRNAs in the network were validated by $\mathrm{qRT}$-PCR in vitro. The mRNA-encoded proteins in the network were used for drug prediction through the Drugbank database, and mRNA-protein-drug networks were constructed. Drug-Gene interaction database and Connectivity map predicted the mRNAs in the network to find linked chemical drugs. The flow chart is illustrated in Figure 1(a).

\section{Materials and Methods}

2.1. Microarray Data Sources. From the Gene Expression Omnibus Database (GEO) (https://www.ncbi.nlm.nih.gov/ 
TABLE 1: Basic information of the two microarray datasets from GEO.

\begin{tabular}{lccc}
\hline Data source & Platform & Series & Sample size (N/T) \\
\hline lncRNA & GPL16956 & GSE124401 & $10 / 10$ \\
mRNA & GPL3050 & GSE3586 & $15 / 13$ \\
\hline
\end{tabular}

N: normal; T: DCM.

geo/), two DCM microarray expression datasets (GSE124401 and GSE3586) were downloaded. The lncRNA dataset included lncRNA expression data in the plasma of 10 normal subjects and ten heart failure (HF) patients diagnosed with dilated cardiomyopathy (DCM). The samples were tested according to GPL16956Agilent-045997 ArraystarhumanlncRNAmicroarrayV3 (ProbeNameVersion) data. The mRNA dataset consisted of mRNA expression data from septal myocardial tissue samples from 13 dilated cardiomyopathy hearts at transplantation and $15 \mathrm{NF}$ donor hearts not transplanted due to visible coronary calcification, tested against data GPL3050HumanUnigene3.1cDNAArray37.5Kv1.0. Table 1 summarizes the necessary information of two DCM microarray expression datasets (GSE124401 and GSE3586).

2.2. Identification of Differentially Expressed Genes. Differential expression analysis was performed on two DCM microarray expression datasets (GSE124401 and GSE3586) using the "limma" package of the R language. The screening criteria for differentially expressed lncRNAs in GSE124401 were I $\log 2 \mathrm{FC} \mid>2$ ( $p$ value $<0.05$ ), and the screening criteria for differentially expressed mRNAs in GSE3586 were |log 2FC $\mid>0.5$ ( $p$ value $<0.05)$.

2.3. Acquisition of Predicted miRNAs and Predicted mRNAs. The differentially expressed lncRNAs described above were predicted by the miRcode database to obtain the corresponding predicted miRNAs, and the predicted miRNAs were further predicted by Targetscan (http://www.targetscan.org/vert_72/), miRTarBase (http://mirtarbase.mbc.nctu.edu.tw/index.html), and miRDB (http://www.mirdb.org/), three databases in combination to get the corresponding predicted mRNAs.

2.4. Cross-Analysis of Differentially Expressed $m R N A$ and Predicted $m R N A$. The intersection of the differentially expressed mRNA with the predicted mRNA described previously was taken to obtain the intersection mRNA, through the Calculate and draw custom Venn diagrams online website (http://bioinformatics.psb.ugent.be/cgibin/liste/Venn/ calculate_venn.htpl), for visualization.

2.5. Construction of Network. The intersected mRNAs and the corresponding predicted miRNAs and differentially expressed IncRNAs together were used to construct the lncRNA-miRNA-mRNA ceRNA network by Cytoscape software. The mRNAs in the ceRNA network were then analyzed using the STRING online website (https://string-db.org/), identified the encoded proteins and constructed proteinprotein interaction (PPI) networks.
2.6. Functional Enrichment Analysis of Differentially Expressed Genes. Using the DAVID online website (https:// david.ncifcrf.gov/home.jsp), GO analysis of differentially expressed mRNAs in the network was performed to assess the cellular component (CC), biological process (BP), and molecular function (MF) of each mRNA. KEGG analysis of differentially expressed mRNAs in the network was performed using the $\mathrm{R}$ language clusterProfiler package to enrich the related pathways. The links between differentially expressed mRNAs and corresponding pathways were visualized with Cytoscape software to construct mRNA-pathway networks.

2.7. Acquisition of Drug Molecules. The proteins encoded by the mRNAs in the ceRNA network mentioned above were accessed through the Drugbank online website (https:/go .drugbank.com/), for drug prediction, and an mRNAprotein-drug network was constructed by Cytoscape software. The mRNAs in the ceRNA network were analyzed by DrugGene interaction database (https://dgidb.genome.wustl.edu/); the drug was predicted directly and visualized with Cytoscape software. The mRNAs in the ceRNA network passed through Connectivity map (https://portals.broadinstitute.org/cmap/), predicted to select drugs with "up score" below -0.645 (i.e., drugs most likely to reverse gene differential expression for DCM), summarized the specific connectivity score of each drug for each mRNA in the specific cell line, and made a heatmap with the $\mathrm{R}$ language pack.

2.8. Cell Culture. The establishment of a cellular model of DCM was performed as previously described [13]. H9c2 cardiomyocytes (Type Culture Collection of Chinese Academy of Sciences, Shanghai, China) were cultured in DMEM supplemented with $10 \%$ FBS in a humidified atmosphere of 5\% $\mathrm{CO}_{2}$ at $37^{\circ} \mathrm{C}$. $\mathrm{H} 9 \mathrm{c} 2$ cardiomyocytes were treated as follows: control cells, in which cells were treated with medium only; doxorubicin treatment group, in which cells were treated with $5 \mu \mathrm{M}$ doxorubicin for 24 hours.

2.9. Real-Time Quantitative PCR. Total RNA was isolated from $\mathrm{H} 9 \mathrm{c} 2$ cells using Trizol reagent (TaKaRa, Japan) according to the manufacturer's instructions. One microgram of RNA was reverse transcribed into cDNA using the Revert Aid First Strand cDNA Synthesis Kit (Thermo, USA). Quantitative RT-PCR was then performed with Pro Taq HS Premix Probe qPCR Kit (Accurate, Hunan, China). The amplification program consisted of one cycle of predenaturation at $95^{\circ} \mathrm{C}$ for $5 \mathrm{~min}, 37$ cycles of denaturation at $95^{\circ} \mathrm{C}$ for $30 \mathrm{~s}$, annealing at $60^{\circ} \mathrm{C}$ for $30 \mathrm{~s}$, and extension at $72^{\circ} \mathrm{C}$ for $10 \mathrm{~min}$. The GAPDH gene was used as an endogenous control gene for normalizing the expression of target genes. Each sample was analyzed in triplicate. Primer sequences are shown in Table 2.

\section{Results}

3.1. Differential Expression Analysis and Prediction of Genes. Seven differentially expressed lncRNAs were obtained from GSE124401 by the screening criteria of $|\log 2 \mathrm{FC}|>2$ ( $p$ value $<0.05)$, and a heatmap was made with the $\mathrm{R}$ language pack 
TABLE 2: The sequences of primers used for qRT-PCR.

\begin{tabular}{lcr}
\hline Gene name & Primer sequences $\left(5^{\prime}-3^{\prime}\right)$ & Annealing temperature \\
\hline AC093817 & F: GCAGGAGAACGAAATTAAGAGACAAG & $60^{\circ} \mathrm{C}$ \\
R: GGCTAGAGGATTATTTGAGACCAGGAT & \\
& F: TATTGCCCATGCCCCTAACTC & $60^{\circ} \mathrm{C}$ \\
Gapdh & R: CTGAAGCCCCCAGACAGTGA & $60^{\circ} \mathrm{C}$ \\
& F: ACAGCAACAGGGTGGTGGAC & \\
\hline
\end{tabular}

F: forward primer; R: reverse primer.

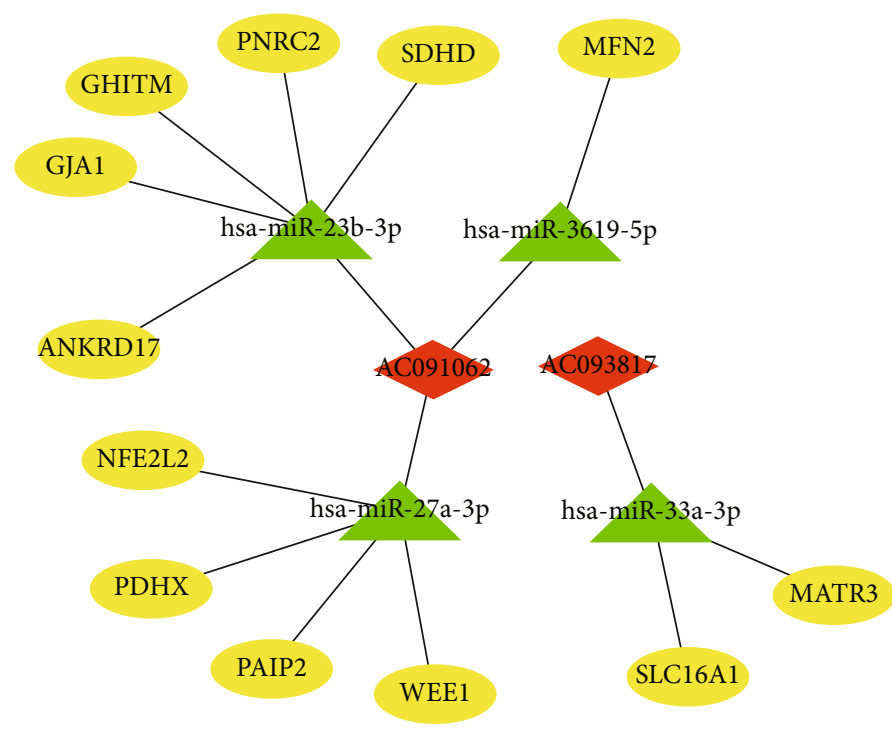

(a)

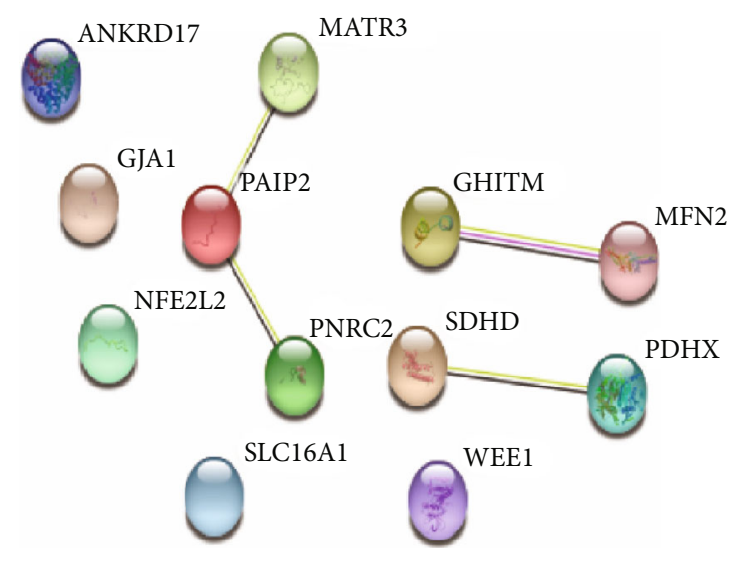

(b)

FIgURE 2: Molecular regulatory interaction network. (a) lncRNA-miRNA-mRNA biomolecular network. Red indicates lncRNA, green indicates miRNA, and yellow indicates mRNA. (b) Protein-protein interaction network of mRNA.

(Figure 1(b)). A screening criterion of $|\log 2 \mathrm{FC}|>0.5$ ( $p$ value $<0.05$ ) yielded 365 differentially expressed mRNAs from GSE3586, and a volcano map was made with the R language pack (Figure 1(c)). The seven differentially expressed lncRNAs resulted in 47 predicted miRNAs by miRcode prediction, and these 47 predicted miRNAs were further predicted by a combination of three databases: Targetscan, miRTarBase, and miRDB resulting in 281 predicted mRNAs.

3.2. Acquisition of Intersection $m R N A$ and Network Construction. From GSE3586, 365 differentially expressed mRNAs were obtained intersecting with 281 predicted mRNAs to take the intersection resulting in 12 intersection mRNAs, visualized with a Wayne diagram (Figure 1(d)). These 12 intersection mRNAs and the corresponding predicted miRNAs and differentially expressed lncRNAs constructed the IncRNA-miRNA-mRNA ceRNA network by Cytoscape software (Figure 2(a)). Twelve mRNAs in the ceRNA network were identified to encode proteins and create a protein interaction (PPI) network through the STRING online website (Figure 2(b)). In the PPI network, protein PAIP2 was connected to the other two proteins, indicating its central regulatory role.
3.3. Enrichment Analysis of mRNAs in the ceRNA Network. GO and KEGG enrichment analyses were performed on the mRNAs in the ceRNA network (Figure 3). GO enrichment analysis showed that "pyruvate metabolic process," "neuronal projection morphogenesis," and "apoptotic process" were enriched in the biological process (BP) classification and "protein binding" was enriched according to the molecular function (MF) classification. Enrichment of "mitochondria," "chromatin," and "mitochondrial outer membrane" was shown in the cellular component (CC) classification (Figures 3(a) and 3(b)); KEGG enrichment analysis showed that differentially expressed mRNAs in the network were mainly involved in signaling pathways such as "Parkinson's disease," "citric acid cycle (TCA cycle)," "amyotrophic lateral sclerosis," and "neurodegeneration" (Figures 3(c) and 3(d)). The mRNA-pathway network was visualized and constructed with Cytoscape software (Figure 4(a)).

3.4. Drug Prediction of $m R N A$ in the ceRNA Network. Twelve mRNA encoded proteins in the ceRNA network predicted drugs through the online website of Drugbank, and four of them got related drugs and constructed the mRNA-proteindrug network (Figure 4(b)). Twelve mRNAs in the ceRNA network predicted drugs directly through the drug-gene 


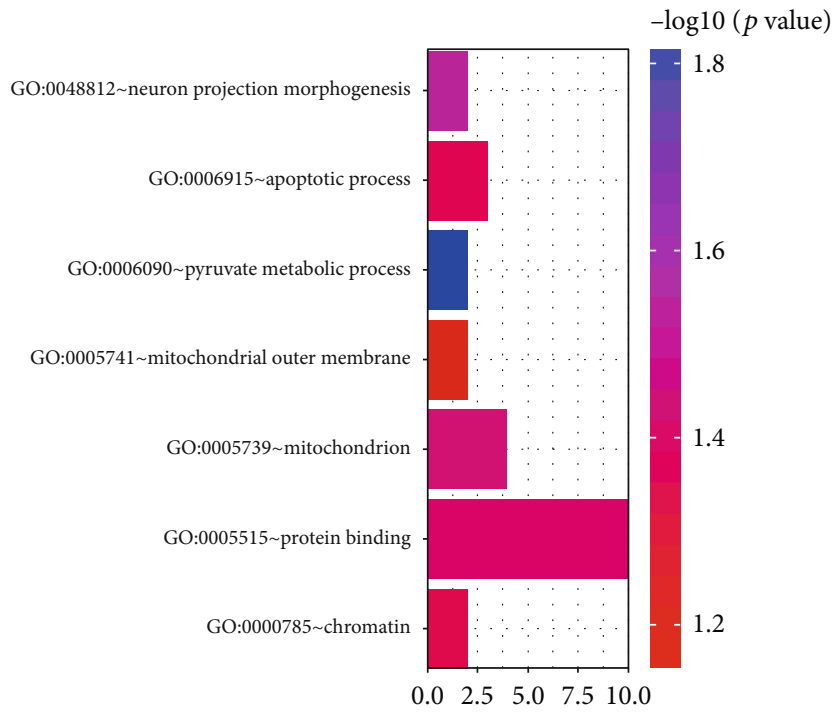

(a)

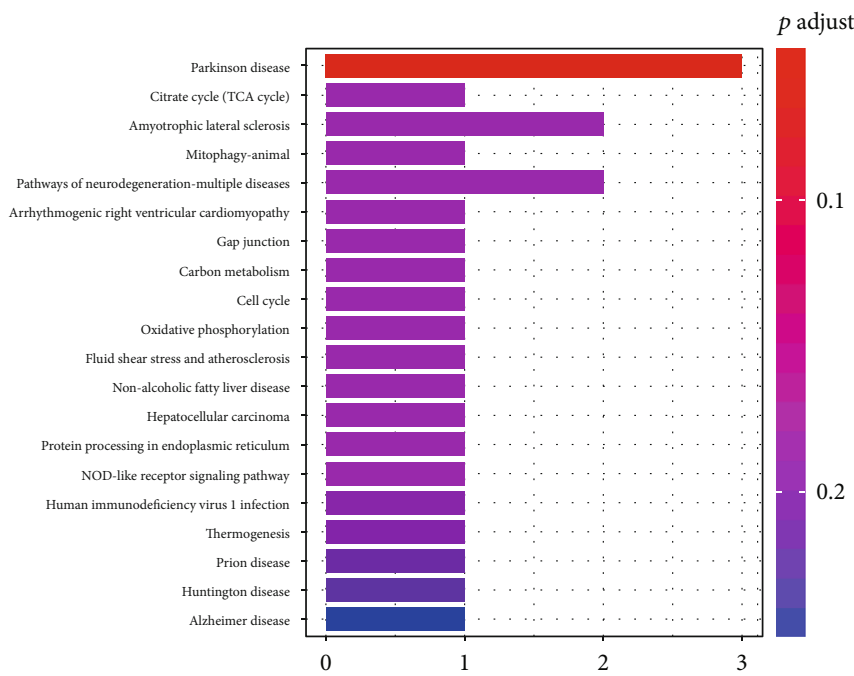

(c)

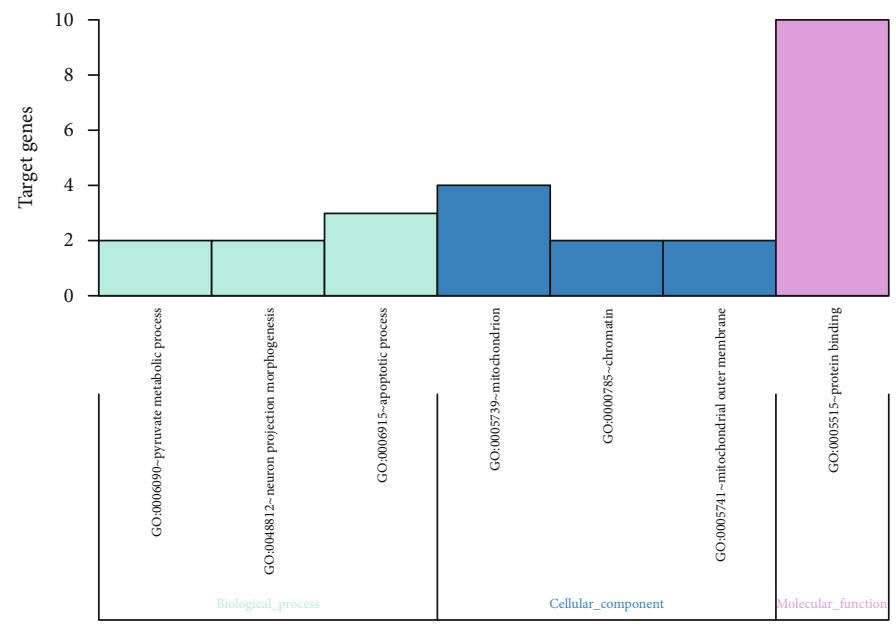

(b)

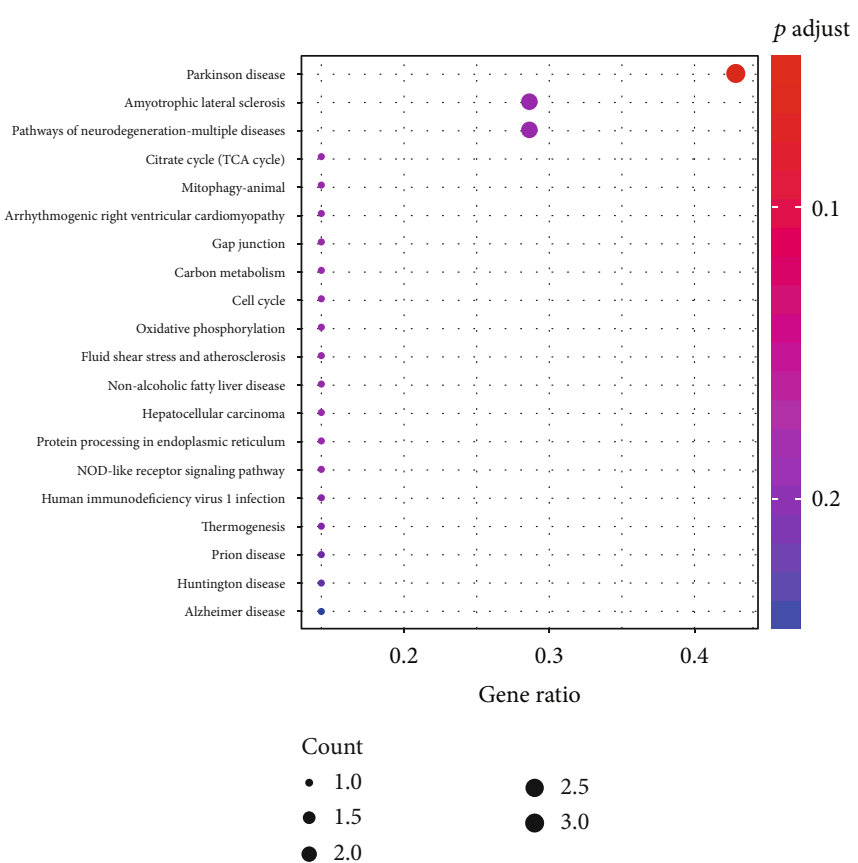

(d)

FIGURE 3: Enrichment analysis of the intersected mRNAs. (a) Bar plot of the GO analysis. (b) Histogram of GO cluster analysis. Target genes are intersected mRNAs. (c) Bar plot of the KEGG analysis. (d) Bubble plot of the KEGG analysis.

interaction database, and five of them got related drugs and constructed the mRNA-drug network (Figure 4(c)). Twelve upregulated mRNAs in the ceRNA network were added to the up tag list. Two downregulated mRNAs were added to the down tag list from GSE3586 differentially expressed mRNAs (setting down downregulated list is only necessary for uploading files, which does not affect drug prediction of the up tag list). The 16 drugs with "up scores" below -0.645 were predicted by the connectivity map. The specific connectivity score of these 16 drugs regarding the expression of 12 upregulated mRNAs was summarized, and the heatmap (Figure $4(\mathrm{~d})$ ) was made with the $\mathrm{R}$ language package.
3.5. Expression of Two lncRNAs in DCM Cells. To validate the expression levels of these two lncRNAs (AC093817 and AC091062) in DCM cells, we validated them by qRT-PCR. The results showed that both AC093817 and AC091062 were significantly overexpressed in DCM cells compared with the normal group (Figure 5). These results are consistent with the results of bioinformatics analysis.

\section{Discussion}

Dilated cardiomyopathy (DCM) is a cardiovascular disease of unknown pathogenesis, one of the leading causes of heart 

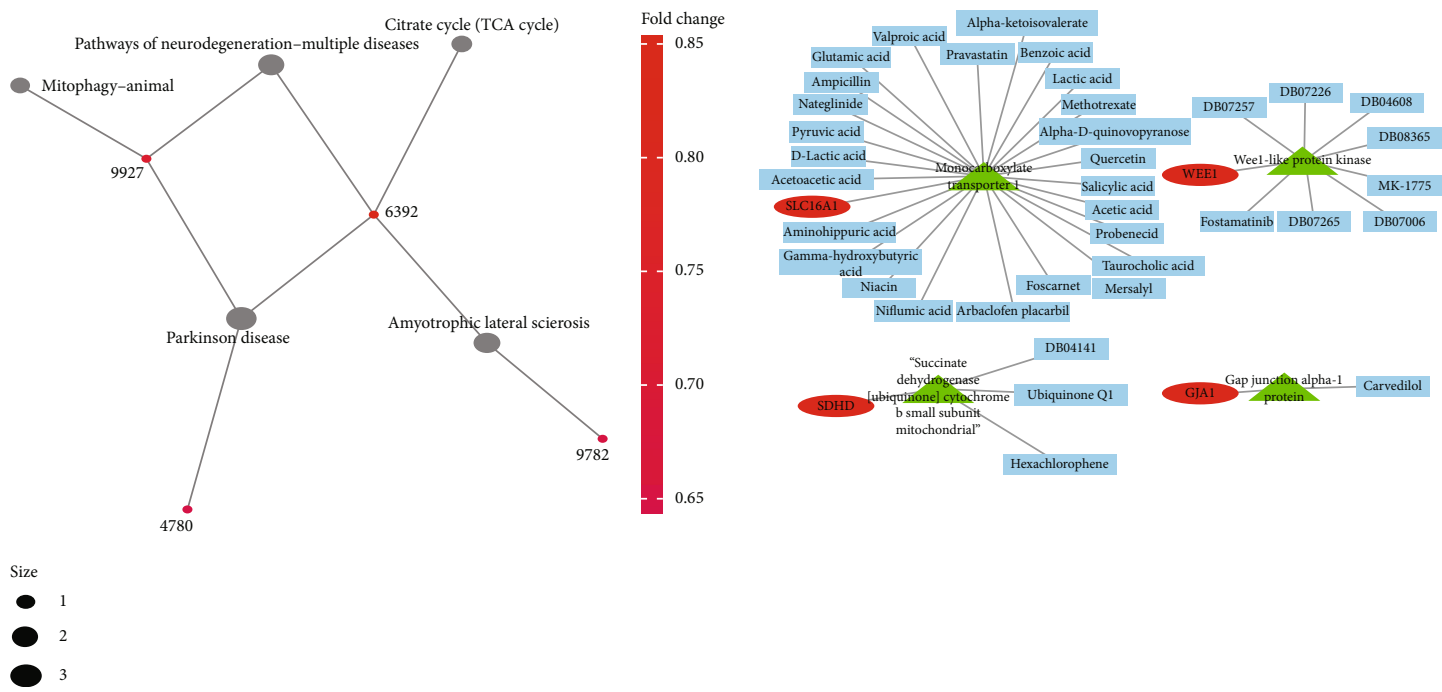

(a)

(b)

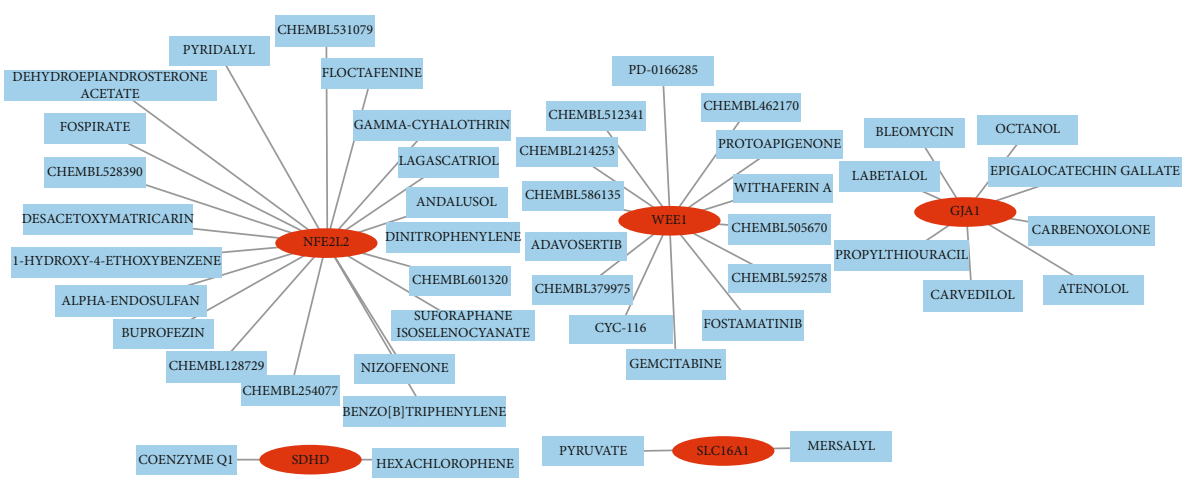

(c)

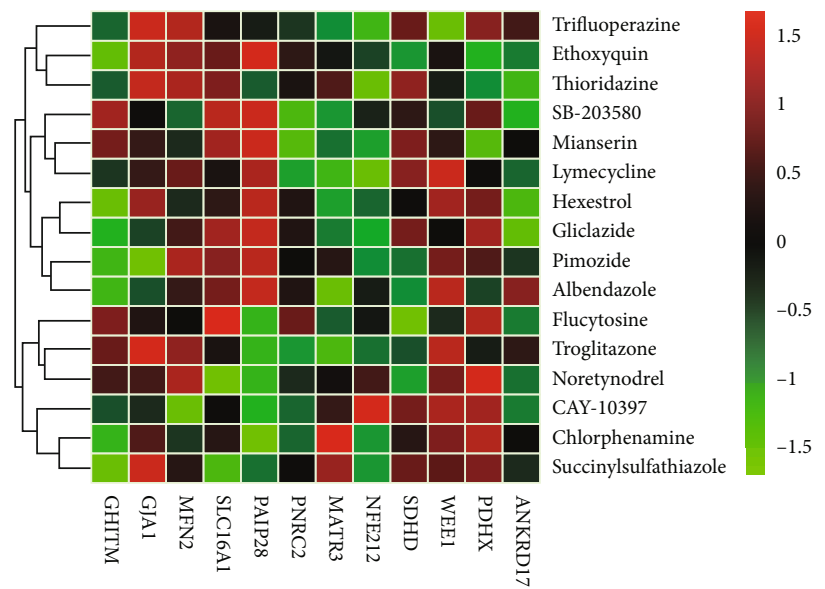

(d)

FIGURE 4: mRNA-pathway network and drug prediction. (a) mRNA-pathway network. Red dots indicate entrezID of mRNA, and black dots are pathways. The mRNA's gene name corresponding to entrezID can be obtained from the Supplementary Table S1. (b) mRNA-proteindrug network. Red ovals represent mRNAs, green triangles represent proteins encoded by mRNAs, and blue rectangles are drugs. Seven of these drugs are indicated by Drugbank-ID because the names are too long, and the drug names corresponding to Drugbank-IDs can be obtained from Supplementary Table S2. (c) mRNA-drug network. Red indicates mRNA, and blue indicates drug. Among them, the 821 drugs corresponding to NFE2L2 showed only the top 20, and all 821 drug names can be obtained in Supplementary Table S3. (d) Heatmap of the effect of drugs on gene expression. Green indicates that the drug can downregulate the gene expression, red marks that the drug can upregulate the gene expression, and black means that the drug and gene expression have little correlation. 


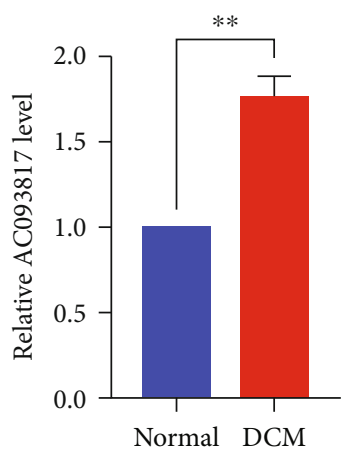

(a)

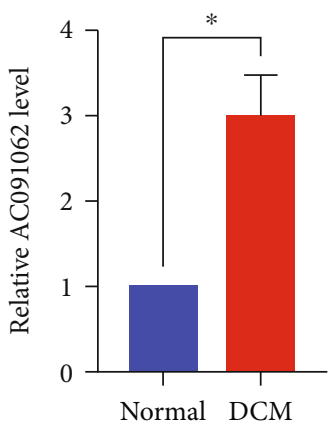

(b)

Figure 5: The expression levels of two lncRNAs involved in DCM were measured by qRT-PCR. $n=3 ; * p<0.05$ and ${ }^{* *} p<0.01$. (a) AC093817 was upregulated in DCM cells. (b) AC091062 was upregulated in DCM cells.

failure. Patients at any stage of the disease are at risk of sudden death. The etiology of dilated cardiomyopathy is very complex and can be regarded as a complex interaction between environmental factors and genetic background. Dilated cardiomyopathy is more common in men than in women, and its prevalence in the general population is estimated at 36 cases per 100,000 [14]. A large number of patients with dilated cardiomyopathy may have an incubation period, clinical manifestations are asymptomatic, the condition is progressively aggravated, severe myocardial failure has developed at the time of diagnosis, which will lead to heart transplantation or even death, and almost $50 \%$ of patients die within five years, which is extremely harmful [14]. Therefore, there is an urgent need to clarify the role of ceRNA networks in the course of DCM and find serum biomarkers and related potential therapeutic agents to provide further support for the diagnosis and treatment of DCM.

Many studies have investigated the mechanism and clinical significance of cardiac lncRNA or even lncRNA-miRNA pairs in DCM in recent years. However, the impact of serum ceRNA networks on DCM in patients with DCM has not been addressed. This study constructed a complete lncRNAmiRNA-mRNA ceRNA network based on lncRNA and mRNA expression profiles for targeted prediction and taking the intersection, providing new ideas for the posttranscriptional level of gene regulation. Drug prediction of mRNAs and their encoded proteins in the ceRNA networks by multiple pharmacogenomics databases may be useful therapeutic molecules in the future. Connectivity map's prediction results showed that all 16 drugs were effective in downregulating the expression of some of these 12 overexpressed mRNAs. However, these 16 drugs also upregulated the expression of some mRNAs to a greater or lesser extent. This may provide support for the active drug structural design of DCM.

The two lncRNAs and 12 mRNAs overexpressed in the network were found for the first time, which are very different from the differentially expressed lncRNAs and mRNAs obtained from previous studies, such as LMNA, FLNC, and TTN, which have been intensely studied in precision medicine for DCM [15]. This may be because previous studies have directly studied differential gene expression from heart samples of DCM patients and controls. Differently, this study newly added the differential gene expression of plasma samples from DCM patients and controls as a starting point on this basis. The new biomarker lncRNAs obtained from the new starting point: AC093817 and AC091062, may provide new ideas for the therapeutic diagnosis of DCM. More importantly, for these two lncRNAs in the ceRNA network, the comparison of qRT-PCR results between doxorubicin-induced derived DCM cells and control further validated that both AC093817 and AC091062 were significantly overexpressed in DCM cells.

The "mitochondria," "pyruvate metabolic process," and "apoptotic process" involved in the overexpressed mRNA in the network may indicate that the cardiac cell metabolism is abnormally active in DCM patients, corresponding to the clinical characteristics of ventricular enlargement in DCM, which is a compensatory mechanism for weakened myocardial contraction and reduced score. The pathway pathways involving overexpressed mRNA not only show cellular metabolic pathways such as citric acid cycle (TCA cycle) and oxidative phosphorylation but also are related to atherosclerosis, viral infection, and even a variety of neurodegenerative diseases (Parkinson's disease, Huntington's disease, Alzheimer's disease, etc.). The diversity of pathways in which it is involved corresponds to its various pathogenic factors such as cardiotoxic compounds, metabolism, rheumatological and endocrine diseases, and cellular infiltration and viral infection [16]. Due to multiple heterogeneous etiologies, dilated cardiomyopathy is an "umbrella" term that describes the final common pathway of different pathogenic processes and gene-environment interactions [17]. A further accurate understanding of the molecular mechanisms and signaling pathways of DCM caused by various etiologies facilitates further subdivision of DCM, which is of great significance for targeted therapy and prognosis of DCM.

In most cases, dilated cardiomyopathy treatment refers to heart failure treatment based on medical treatment with angiotensin-converting enzyme inhibitors (angiotensin receptor blockers or angiotensin receptor/neprilysin inhibitors, if applicable), beta-blockers, and mineralocorticoid receptor antagonists according to current guidelines for heart failure [16]. Different from the previous ideas of symptomatic treatment with drugs to delay disease progression, this study selects the corresponding drugs through multiple 
pharmacogenomics databases based on the new thinking of targeted therapy for specific disease mechanisms to reverse the disease process, targeting the 12 mRNAs overexpressed in the network and their encoded proteins, and the mechanistic analysis of the downregulation of mRNA expression and inhibition of proteins by these drugs in the future will further reveal the common pathways of their effects, which can be used as the starting point for subsequent studies.

Validating effective RNA treatment strategies in a number of cancer treatment studies [18-24] can also be used as an adjuvant treatment for DCM. In the future, we can construct a nanotargeted therapeutic system by designing miRNA overexpression vector gene drugs together with chemical drugs through rational encapsulation of nanomaterials such as cationic polymers and liposomes and connecting cardiac-targeting ligands in the outer layer [25-27]. With the rapid development of RNA nanotechnology, we can also construct RNAs with specific folded structures and spatial conformations to achieve downregulation of overexpressed mRNAs, such as miRNAs and siRNAs with specific structures and functions. Of course, this requires a lot of basic analysis and experimental validation [28-30].

\section{Conclusion}

In conclusion, in this study, we constructed a complete DCM differentially expressed IncRNA-miRNA-mRNA ceRNA network, found that AC093817 and AC091062 are potential biomarkers of dilated cardiomyopathy, and proposed potential therapeutic agents that can be used for this disease by integrating and analyzing microarray gene expression data, providing support for possible mechanisms and drug development of dilated cardiomyopathy.

\section{Data Availability}

The data used to support the findings of this study are available in the Gene Expression Omnibus (GEO) repository (https://www.ncbi.nlm.nih.gov/gds).

\section{Conflicts of Interest}

The authors declare that there is no conflict of interest regarding the publication of this paper.

\section{Acknowledgments}

This study was supported by the Scientific Research Project of Hunan Health Commission (No. 202102041763 and No. 20200985), Natural Science Foundation of Changsha (No. 202045504), and Hunan Cancer Hospital Climb Plan (No. 2020QH001).

\section{Supplementary Materials}

Supplementary 1. Table S1: the gene name corresponding to the entrezID of the mRNA on Figure 4(a).

Supplementary 2. Table S2: the drug name corresponding to the Drugbank-ID of the drug on Figure 4(b).
Supplementary 3. Table S3: all 821 drug names corresponding to NFE2L2 on Figure 4(c).

\section{References}

[1] P. Elliott, B. Andersson, E. Arbustini et al., "Classification of the cardiomyopathies: a position statement from the European Society of Cardiology Working Group on Myocardial and Pericardial Diseases," European Heart Journal, vol. 29, no. 2, pp. 270-276, 2007.

[2] Y. M. Pinto, P. M. Elliott, E. Arbustini et al., "Proposal for a revised definition of dilated cardiomyopathy, hypokinetic non-dilated cardiomyopathy, and its implications for clinical practice: a position statement of the ESC working group on myocardial and pericardial diseases," European Heart Journal, vol. 37, no. 23, pp. 1850-1858, 2016.

[3] X. Cheng and H. Jiang, "Long non-coding RNA HAND2-AS1 downregulation predicts poor survival of patients with endstage dilated cardiomyopathy," The Journal of International Medical Research, vol. 47, no. 8, pp. 3690-3698, 2019.

[4] E. K. Robinson, S. Covarrubias, and S. Carpenter, "The how and why of lncRNA function: an innate immune perspective," Biochimica et Biophysica Acta (BBA) - Gene Regulatory Mechanisms, vol. 1863, no. 4, article 194419, 2020.

[5] Y. Zhang, M. Zhang, W. Xu, J. Chen, and X. Zhou, "The long non-coding RNA H19 promotes cardiomyocyte apoptosis in dilated cardiomyopathy," Oncotarget, vol. 8, no. 17, pp. 28588-28594, 2017.

[6] H. Li, C. Chen, J. Fan et al., "Identification of cardiac long noncoding RNA profile in human dilated cardiomyopathy," Cardiovascular Research, vol. 114, no. 5, pp. 747-758, 2018.

[7] F. Lin, X. Gong, P. Yu et al., "Distinct circulating expression profiles of long noncoding RNAs in heart failure patients with ischemic and nonischemic dilated cardiomyopathy," Frontiers in Genetics, vol. 10, p. 1116, 2019.

[8] P. Wang, H. Fu, J. Cui, and X. Chen, "Differential lncRNAmRNA co-expression network analysis revealing the potential regulatory roles of lncRNAs in myocardial infarction," Molecular Medicine Reports, vol. 13, no. 2, pp. 1195-1203, 2016.

[9] X. Luo, P. Luo, and Y. Zhang, "Identification of differentially expressed long non-coding RNAs associated with dilated cardiomyopathy using integrated bioinformatics approaches," Drug Discov Ther, vol. 14, no. 4, pp. 181-186, 2020.

[10] X. Zhang, X. Nie, S. Yuan et al., "Circulating long non-coding RNA ENST00000507296 is a prognostic indicator in patients with dilated cardiomyopathy," Mol Ther Nucleic Acids, vol. 16, pp. 82-90, 2019.

[11] Z. Qiu, B. Ye, L. Yin, W. Chen, Y. Xu, and X. Chen, "Downregulation of AC061961.2, LING01-AS1, and RP11-13E1.5 is associated with dilated cardiomyopathy progression," Journal of Cellular Physiology, vol. 234, no. 4, pp. 4460-4471, 2019.

[12] L. Tao, L. Yang, X. Huang, F. Hua, and X. Yang, "Reconstruction and analysis of the lncRNA-miRNA-mRNA network based on competitive endogenous RNA reveal functional lncRNAs in dilated cardiomyopathy," Frontiers in Genetics, vol. 10, p. 1149, 2019.

[13] Y. Xia, Z. Chen, A. Chen et al., "LCZ696 improves cardiac function via alleviating Drp1-mediated mitochondrial dysfunction in mice with doxorubicin-induced dilated cardiomyopathy," Journal of Molecular and Cellular Cardiology, vol. 108, pp. 138-148, 2017. 
[14] H. Mahmaljy, V. S. Yelamanchili, and M. Singhal, "Dilated cardiomyopathy," in StatPearls, StatPearls Publishing LLC, Treasure Island (FL), 2020.

[15] A. Morales, D. D. Kinnamon, E. Jordan et al., "Variant interpretation for dilated cardiomyopathy: refinement of the American College of Medical Genetics and Genomics/ClinGen Guidelines for the DCM Precision Medicine Study," Circulation: Genomic and Precision Medicine, vol. 13, no. 2, article e002480, 2020.

[16] A. Hänselmann, C. Veltmann, J. Bauersachs, and D. Berliner, "Dilated cardiomyopathies and non-compaction cardiomyopathy," Herz, vol. 45, no. 3, pp. 212-220, 2020.

[17] M. Merlo, A. Cannatà, and G. Sinagra, "Dilated cardiomyopathy: a paradigm of revolution in medicine," Journal of Clinical Medicine, vol. 9, no. 11, article 3385, 2020.

[18] R. Esteve-Puig, A. Bueno-Costa, and M. Esteller, "Writers, readers and erasers of RNA modifications in cancer," Cancer Letters, vol. 474, pp. 127-137, 2020.

[19] J. Desterro, P. Bak-Gordon, and M. Carmo-Fonseca, "Targeting mRNA processing as an anticancer strategy," Nature Reviews. Drug Discovery, vol. 19, no. 2, pp. 112-129, 2020.

[20] Y. Xie, Y. Hang, Y. Wang et al., "Stromal modulation and treatment of metastatic pancreatic cancer with local intraperitoneal triple miRNA/siRNA nanotherapy," ACS Nano, vol. 14, no. 1, pp. 255-271, 2020.

[21] C. M. Guo, S. Q. Liu, and M. Z. Sun, "miR-429 as biomarker for diagnosis, treatment and prognosis of cancers and its potential action mechanisms: a systematic literature review," Neoplasma, vol. 67, no. 2, pp. 215-228, 2020.

[22] R. Li, J. Jiang, H. Shi, H. Qian, X. Zhang, and W. Xu, "CircRNA: a rising star in gastric cancer," Cellular and Molecular Life Sciences, vol. 77, no. 9, pp. 1661-1680, 2020.

[23] J. Liu and B. Guo, "RNA-based therapeutics for colorectal cancer: updates and future directions," Pharmacological Research, vol. 152, article 104550, 2020.

[24] K. F. Pirollo, A. Rait, Q. Zhou et al., "Materializing the potential of small interfering RNA via a tumor-targeting nanodelivery system," Cancer Research, vol. 67, no. 7, pp. 2938-2943, 2007.

[25] L. Li, J. Hou, X. Liu et al., "Nucleolin-targeting liposomes guided by aptamer AS1411 for the delivery of siRNA for the treatment of malignant melanomas," Biomaterials, vol. 35, no. 12, pp. 3840-3850, 2014.

[26] S. Guan and J. Rosenecker, "Nanotechnologies in delivery of mRNA therapeutics using nonviral vector- based delivery systems," Gene Therapy, vol. 24, no. 3, pp. 133-143, 2017.

[27] E. Keles, Y. Song, D. Du, W. J. Dong, and Y. Lin, "Recent progress in nanomaterials for gene delivery applications," Biomaterials Science, vol. 4, no. 9, pp. 1291-1309, 2016.

[28] H. Ohno, S. Akamine, and H. Saito, "RNA nanostructures and scaffolds for biotechnology applications," Current Opinion in Biotechnology, vol. 58, pp. 53-61, 2019.

[29] S. Kobori, Y. Nomura, and Y. Yokobayashi, "Self-powered RNA nanomachine driven by metastable structure," Nucleic Acids Research, vol. 47, no. 11, pp. 6007-6014, 2019.

[30] D. Jedrzejczyk, E. Gendaszewska-Darmach, R. Pawlowska, and A. Chworos, "Designing synthetic RNA for delivery by nanoparticles," Journal of Physics: Condensed Matter, vol. 29, no. 12, article 123001, 2017. 